\title{
Análise das Discordâncias Diagnósticas dos Exames Citopatológicos do Programa de Monitoramento Externo de Qualidade no Estado de São Paulo, Brasil, 2000-2010
} Analysis of the Diagnostic Disagreements in Cytopathologic Exams from the External Quality Assurance Program in the State of São Paulo, Brazil, 2000-2010 Análisis de las Discordancias Diagnósticas de Pruebas Citopatológicas del Programa de Supervisión Externa de Calidad en el Estado de São Paulo, Brasil, 2000-2010

\author{
Daniela Etlinger'; Sonia Maria Miranda Pereira ${ }^{2}$; Yuriko Ito Sakai ${ }^{3}$; Luzia Setuko Umeda Yamamoto ${ }^{4}$; Silvia D’Andretta Iglezias ${ }^{5}$; Monique Camila \\ Basso $^{6}$; Celso di Loreto ${ }^{7}$
}

\section{Resumo}

Introduçáo: O Programa de Monitoramento Externo de Qualidade (MEQ) dos exames citopatológicos cervicovaginais realizados pelo Sistema Único de Saúde (SUS) foi proposto pelo Ministério da Saúde (MS) para melhorar o desempenho dos laboratórios de citologia da Rede Pública de Saúde. Objetivo: Avaliar as discordâncias diagnósticas dos exames citopatológicos cervicovaginais submetidos à revisão pelo Programa de MEQ do Estado de São Paulo, no período de 2000 a 2010. Método: Avaliação comparativa retrospectiva dos diagnósticos emitidos pelos lab-SUS e dos diagnósticos de revisão dos exames citopatológicos cervicovaginais. Resultados: Das 123.002 amostras revisadas, $16.581(13,48 \%)$ apresentaram discordância diagnóstica, sendo que, em $14.313(11,64 \%)$ casos, houve divergência entre a conduta adotada e a preconizada pelo Instituto Nacional de Câncer José Alencar Gomes da Silva (INCA). Foram considerados exames falso-positivos 2.530 (2,06\%) e falso-negativos 2.154 (1,75\%). A sensibilidade foi de $92,02 \%$ e especificidade de $96,49 \%$. Houve concordância boa entre os diagnósticos originais e de revisão ( $k a p p a=0,77)$. Conclusáo: Além do conjunto de atividades básicas propostas pelo MS, o MEQ do Estado de São Paulo destaca as açôes educativas implementadas, adotadas para promover a educação continuada no sentido da uniformização de critérios citomorfológicos e a consequente redução dos resultados falso-negativos e falso-positivos.

Palavras-chave: Controle de Qualidade; Citodiagnóstico; Neoplasias do Colo do Útero/diagnóstico; Neoplasias do Colo do Útero/patologia; Programas de Rastreamento; Prevenção de Câncer de Colo Uterino

\footnotetext{
${ }^{1}$ Biomédica. Pesquisador Científico III do Laboratório de Citologia Oncótica. Núcleo de Anatomia Patológica do Instituto Adolfo Lutz - Central. E-mail: dani_etlinger@yahoo.com.br.

${ }^{2}$ Biomédica. Pesquisador Científico VI do Laboratório de Citologia Oncótica. Diretora do Núcleo de Anatomia Patológica do Instituto Adolfo Lutz - Central. E-mail: mirandasmp@yahoo.com.br.

${ }^{3}$ Biomédica. Pesquisador Científico VI do Laboratório de Citologia Oncótica. Núcleo de Anatomia Patológica do Instituto Adolfo Lutz - Central. E-mail: yursakai@gmail.com.

${ }^{4}$ Biomédica. Pesquisador Científico VI do Laboratório de Citologia Oncótica. Núcleo de Anatomia Patológica do Instituto Adolfo Lutz - Central. E-mail: luyamamot@hotmail.com.

${ }^{5}$ Médico Patologista do Laboratório de Citologia Oncótica. Núcleo de Anatomia Patológica do Instituto Adolfo Lutz - Central. E-mail: silviaiglezias@uol.com.br. ${ }^{6}$ Médico Patologista do Laboratório de Citologia Oncótica. Núcleo de Anatomia Patológica do Instituto Adolfo Lutz - Central. E-mail: moniquebasso@uol.com.br. ${ }^{7}$ Médico Patologista do Laboratório de Citologia Oncótica. Núcleo de Anatomia Patológica do Instituto Adolfo Lutz - Central. E-mail: celsodiloreto@gmail.com. Endereço para correspondência: Daniela Etlinger. Avenida Dr. Arnaldo, 355 - 7º andar. Cerqueira César. CEP: 01246-902. Sáo Paulo (SP), Brasil.
} 


\section{INTRODUÇÃO}

O câncer do colo do útero ainda é um sério problema de Saúde Pública pelas altas taxas de prevalência e mortalidade ${ }^{1}$. No Brasil, o Instituto Nacional de Câncer José Alencar Gomes da Silva (INCA) estima, para 2012, 17.540 novos casos de câncer do colo do útero, sendo que para o Estado de São Paulo (SP) a taxa estimada é de $2.880 \operatorname{casos}^{2}$. O rastreamento e detecção precoce das lesōes pré-neoplásicas são as principais ferramentas para reduzir a mortalidade, sendo o exame de Papanicolaou o método preconizado e amplamente utilizado na Rede Pública do país ${ }^{1}$.

De acordo com dados da literatura mundial, o exame de Papanicolaou foi fundamental para a redução da mortalidade desta doença devido à alta especificidade (98\%); porém, a baixa sensibilidade $(51 \%)$ resulta em elevadas taxas de incidência e mortalidade nos países menos desenvolvidos ${ }^{3-4}$. Para garantir a efetividade dos programas de rastreamento, são necessárias açóes voltadas para a organização do sistema de informação, qualidade dos diagnósticos, bem como a garantia de seguimento das pacientes ${ }^{1}$.

Em 2000, o Ministério da Saúde (MS) preconizou que todos os laboratórios que realizam exames citopatológicos para o SUS deveriam submeter-se ao Programa de Monitoramento Externo de Qualidade (MEQ), com o intuito de avaliar o desempenho diagnóstico e obter fomentos para educaçấo continuada dos profissionais envolvidos ${ }^{5-6}$.

A Resolução SS-116 de 27/07/2000 instituiu a Fundação Oncocentro de São Paulo (FOSP) e o Instituto Adolfo Lutz de São Paulo (IAL), como responsáveis pelo MEQ no Estado de SP, sendo a FOSP responsável pelas atividades administrativas e o IAL responsável pelas atividades técnico-científicas. Ao longo desses anos, este trabalho vem sendo desenvolvido dentro das normas propostas pelo INCA/MS $\mathrm{MS}^{5-7}$.

O objetivo do estudo foi avaliar as discordâncias diagnósticas dos exames citopatológicos cervicovaginais submetidos à revisão pelo Programa de MEQ do Estado de São Paulo no período de 2000 a 2010.

\section{MÉTODO}

O presente estudo consiste em avaliação comparativa retrospectiva dos diagnósticos emitidos pelos lab-SUS e dos diagnósticos de revisão dos exames citopatológicos cervicovaginais submetidos ao MEQ no período de 2000 a 2010. Todas as amostras cervicovaginais foram processadas pelos lab-SUS e diagnosticadas segundo a Nomenclatura Brasileira para Laudos Citopatológicos ${ }^{8}$. O projeto foi aprovado pelo Comitê de Ética em Pesquisa do Instituto Adolfo Lutz (protocolo $n^{\circ} 23.680$ ).

Para a revisão são selecionados anualmente, aleatoriamente pelo SISCOLO, $10 \%$ dos exames realizados por cada laboratório/mês, de acordo com os critérios de seleção recomendados, incluindo todos os casos positivos (atipias de significado indeterminado, lesões intraepiteliais de baixo e alto graus, carcinomas escamosos e adenocarcinomas); todos os insatisfatórios e no mínimo $5 \%$ dos exames negativos ${ }^{9}$. Os lab-SUS encaminharam as lâminas selecionadas para a FOSP, responsável pelo processo administrativo, que as enviou ao Laboratório de Citologia Oncótica do Instituto Adolfo Lutz - Central (LCO-IAL), onde foi realizada a avaliação pré-analítica (qualidade da confecção da amostra, fixação, coloração, montagem e contaminação das amostras) e analítica das amostras. A revisão dos casos foi realizada com conhecimento prévio dos diagnósticos originais pelos examinadores, conforme as diretrizes do MEQ. Para análise dos dados, foi utilizado o índice estatístico kappa ponderado, cálculo de discordância entre os diagnósticos dos lab-SUS e LCO-IAL, cálculo de sensibilidade e especificidade e a frequência de exames falso-negativos (FN) e falso-positivos (FP) no período, considerando o diagnóstico de revisão o padrão ouro para as análises.

Entre 2000 e 2010, o LCO-IAL revisou 123.002 lâminas provenientes de 140 laboratórios. Nos anos de 2005 e 2006, não foi realizada revisão devido à atualização da nomenclatura brasileira para os laudos citopatológicos no SISCOLO.

Para a análise, foram considerados os diagnósticos: insatisfatório, negativo (dentro dos limites da normalidade e inflamação), ASC-US (células atípicas escamosas de significado indeterminado, possivelmente não neoplásicas), ASC-H (células atípicas escamosas de significado indeterminado, não se pode afastar lesão intraepitelial de alto grau), LSIL (lesão escamosa intraepitelial de baixo grau), HSIL (lesão escamosa intraepitelial de alto grau), HSILmi (lesão escamosa intraepitelial de alto grau não podendo excluir microinvasão), $\mathrm{CA}$ (carcinoma epidermoide invasor), AGC-US (células atípicas glandulares de significado indeterminado, possivelmente não neoplásicas), AGC-H (células atípicas glandulares de significado indeterminado, não se pode afastar lesão intraepitelial de alto grau), adeno in situ (adenocarcinoma in situ), adenoinvasor (adenocarcinoma invasor), AOCUS (células atípicas de origem indefinida de significado indeterminado possivelmente não neoplásicas) e AOC-H (células atípicas de origem indefinida de significado indeterminado não se pode afastar lesão intraepitelial de alto grau). A alteraçáo de conduta clínica para a paciente foi considerada para avaliar a concordância diagnóstica de acordo com as recomendaçóes do INCA/MS ${ }^{8}$ (Figura 1). 




\section{CONDUTA PRECONIZADA}

Figura 1. Diagnósticos agrupados de acordo com conduta clínica, sendo considerada discordância diagnóstica aquela que acarreta modificação da conduta

\section{RESULTADOS}

Das 123.002 lâminas citológicas revisadas, o LCOIAL classificou como insatisfatórios $12,55 \%$ (15.431) amostras, negativo 64,49\% (79.327), ASC-US 10,02\% (12.323), ASC-H 0,68\% (839), LSIL 8,05\% (9.907), HSIL 2,96\% (3.642), HSIL mi 0,13\% (164), CA 0,29\% (361), AGC-US 0,67\% (827), AGC-H 0,09\% (112), adeno in situ $0,02 \%$ (20), adenoinvasor $0,02 \%$ (26), AOC-US 0,01\% (13) e AOC-H 0,01\% (10).

Os lab-SUS classificaram como insatisfatórios 7,58\% $(9,319)$ amostras, negativo 68,59\% (84.367), ASC-US 10,06\% (12.369), ASC-H 0,79\% (971), LSIL 8,44\% (10.387), HSIL 2,98\% (3.670), HSIL mi 0,13\% (157),
CA 0,28\% (349), AGC-US 0,77\% (950), AGC-H 0,12\% (145), Adeno in situ $0,02 \%$ (24), adenoinvasor $0,13 \%$ (163), AOC-US 0,07\% (83) e AOC-H 0,04\% (45) e outras neoplasias $0,002 \%$ (3).

A discordância de diagnósticos entre os lab-SUS e o LCO-IAL foi de 13,48\% (16.581). A maior discordância foi observada entre os casos de HSIL mi $(61,59 \%)$ e a menor discordância entre os exames classificados como negativos (4,19\%) (Tabela 1$)$.

O LCO-IAL classificou como negativo 2.530 $(2,06 \%)$ amostras diagnosticadas pelos lab-SUS como alteraçôes epiteliais (FP), sendo que 1.798 (1,46\%) foram originalmente classificadas como ASC-US, 37 (0,03\%) ASC-H, 470 (0,38\%) LSIL, 64 (0,05\%) HSIL, 4

Tabela 1.Distribuição dos diagnósticos citopatológicos do lab-SUS e do LCO-IAL submetidos à revisão pelo MEQ, no período de 2000 a 2010

\begin{tabular}{|c|c|c|c|c|c|c|c|c|c|c|c|c|c|c|c|c|}
\hline \multirow{2}{*}{$\begin{array}{c}\text { Diagnóstico } \\
\text { Lab-SUS }\end{array}$} & \multirow{2}{*}{ Insatisf. } & \multirow{2}{*}{ Negativo } & \multicolumn{6}{|c|}{ Células escamosas } & \multicolumn{4}{|c|}{ Células glandulares } & \multicolumn{2}{|c|}{ Orig. indef. } & \multicolumn{2}{|c|}{ TOTAL } \\
\hline & & & $\begin{array}{l}\text { ASC- } \\
\text { US }\end{array}$ & ASC-H & LSIL & HSIL & $\begin{array}{c}\text { HSIL } \\
\text { mi }\end{array}$ & CA & $\begin{array}{c}\text { AGC- } \\
\text { US }\end{array}$ & AGC-H & $\begin{array}{l}\text { Adeno } \\
\text { in situ }\end{array}$ & Adenoinvasor & $\begin{array}{l}\text { AOC- } \\
\text { US }\end{array}$ & AOC-H & N & $\%$ \\
\hline Insatisf. & 8.423 & 796 & 68 & 6 & 14 & 3 & - & 2 & 6 & - & 1 & - & - & - & 9.319 & 7,58 \\
\hline Negativo & 6.212 & 76.001 & 1.447 & 70 & 417 & 71 & 3 & 9 & 125 & 6 & 1 & 5 & - & - & 84.367 & 68,59 \\
\hline ASC-US & 429 & 1.798 & 9.142 & 90 & 701 & 152 & 0 & 18 & 38 & 1 & - & - & - & - & 12.369 & 10,06 \\
\hline ASC-H & 30 & 37 & 292 & 498 & 29 & 68 & 2 & 5 & 3 & 6 & 1 & - & - & - & 971 & 0,79 \\
\hline LSIL & 230 & 470 & 956 & 41 & 8.395 & 276 & 1 & 2 & 15 & 1 & - & - & - & - & 10.387 & 8,44 \\
\hline HSIL & 54 & 64 & 120 & 54 & 294 & 2.937 & 86 & 45 & 7 & 5 & 1 & 3 & - & - & 3.670 & 2,98 \\
\hline HSIL mi & 3 & 4 & 1 & 7 & 3 & 51 & 63 & 25 & - & - & - & - & - & - & 157 & 0,13 \\
\hline CA & 24 & 9 & 15 & 5 & 4 & 33 & 7 & 249 & - & 1 & 1 & 1 & - & - & 349 & 0,28 \\
\hline AGC-US & 18 & 112 & 155 & 24 & 7 & 23 & - & 1 & 594 & 14 & 2 & - & - & - & 950 & 0,77 \\
\hline AGC-H & 1 & 8 & 12 & 21 & - & 10 & - & - & 20 & 72 & - & - & - & 1 & 145 & 0,12 \\
\hline Adeno in situ & 2 & 2 & - & - & 1 & 4 & 1 & 2 & 2 & - & 9 & 1 & - & - & 24 & 0,02 \\
\hline Adenoinvasor & 1 & 11 & 67 & 3 & 39 & 9 & 1 & 2 & 7 & 3 & 4 & 16 & - & - & 163 & 0,13 \\
\hline AOC-US & 3 & 15 & 39 & 4 & - & - & - & - & 8 & 1 & - & - & 13 & - & 83 & 0,07 \\
\hline $\mathrm{AOC}-\mathrm{H}$ & 1 & 0 & 8 & 16 & 3 & 3 & - & 1 & 2 & 2 & - & - & - & 9 & 45 & 0,04 \\
\hline Outras neoplasias & - & - & 1 & - & - & 2 & - & - & - & - & - & - & - & - & 3 & 0,002 \\
\hline \multirow[t]{2}{*}{ TOTAL } & 15.431 & 79.327 & 12.323 & 839 & 9.907 & 3.642 & 164 & 361 & 827 & 112 & 20 & 26 & 13 & 10 & 123.002 & \\
\hline & 12,55 & 64,49 & 10,02 & 0,68 & 8,05 & 2,96 & 0,13 & 0,29 & 0,67 & 0,09 & 0,02 & 0,02 & 0,01 & 0,01 & & \\
\hline \multirow[t]{2}{*}{ Discordância } & 7.008 & 3.326 & 3.181 & 341 & 1.512 & 705 & 101 & 112 & 233 & 40 & 11 & 10 & 0 & 1 & 16.581 & \\
\hline & 45,42 & 4,19 & 25,81 & 40,64 & 15,26 & 19,36 & 61,59 & 31,02 & 28,17 & 35,71 & 55,00 & 38,46 & 0,00 & 10,00 & 13,48 & \\
\hline
\end{tabular}


(0,003\%) HSIL mi, 9 (0,01\%) CA, 112 (0,09\%) AGC-US, $8(0,01 \%)$ AGC-H, $2(0,002 \%)$ adeno in situ, $11(0,01 \%)$ adenoinvasor e $15(0,01 \%)$ AOC-US.

Dos exames diagnosticados pelos lab-SUS como negativos, após a revisão o LCO-IAL detectou 2.154 $(1,75 \%)$ casos com alteraçóes epiteliais (FN), sendo que $1.447(1,18 \%)$ foram reclassificados como ASC-US, 70 (0,06\%) ASC-H, 417 (0,34\%) LSIL, 71 (0,06\%) HSIL, $3(0,002 \%)$ HSIL mi, 9 (0,01\%) CA, 125 (0,10\%) AGC-US, 6 $(0,005 \%)$ AGC-H, $1(0,001 \%)$ adeno in situ e $5(0,004 \%)$ adenoinvasor.

Houve alteração de conduta clínica preconizada em 86,32\% (14.313) dos casos com diagnóstico discordantes (16.581). Destes, 3.036 (2,48\%) deveriam ter repetido a citologia em 6 meses, $943(0,77 \%)$ deveriam ter realizado colposcopia, $7.008(5,70 \%)$ deveriam ter realizado nova coleta de material cervicovaginal e $3.326(2,70 \%)$ deveriam ter seguido a rotina de rastreamento (Tabela 2).

Considerando o resultado de revisão do LCO-IAL como padrão ouro, a sensibilidade foi de $92,02 \%$ e especificidade de $96,49 \%$. O cálculo do kappa ponderado mostrou concordância boa $(0,77)$ entre os lab-SUS e o LCO-IAL.

Tabela 2. Distribuição das divergências entre a conduta preconizada pelo Ministério da Saúde e a conduta adotada de acordo com o laudo emitido pelo lab-SUS, no período de 2000 a 2010

\begin{tabular}{|c|c|c|c|}
\hline \multirow{2}{*}{$\begin{array}{l}\text { Conduta } \\
\text { adotada }\end{array}$} & \multirow{2}{*}{$\begin{array}{c}\text { Conduta } \\
\text { preconizada }\end{array}$} & \multicolumn{2}{|c|}{ TOTAL } \\
\hline & & $\mathbf{N}$ & $\%$ \\
\hline \multirow{3}{*}{$\begin{array}{l}\text { Seguir } \\
\text { rotina de } \\
\text { rastreamento }\end{array}$} & $\begin{array}{l}\text { Repetir em } 6 \\
\text { meses }\end{array}$ & 1.864 & 1,52 \\
\hline & Colposcopia & 290 & 0,24 \\
\hline & Nova coleta & 6.212 & 5,05 \\
\hline \multirow{3}{*}{$\begin{array}{l}\text { Repetir em } 6 \\
\text { meses }\end{array}$} & $\begin{array}{l}\text { Seguir rotina de } \\
\text { rastreamento }\end{array}$ & 2.268 & 1,84 \\
\hline & Colposcopia & 635 & 0,52 \\
\hline & Nova coleta & 659 & 0,54 \\
\hline \multirow{3}{*}{ Colposcopia } & $\begin{array}{l}\text { Seguir rotina de } \\
\text { rastreamento }\end{array}$ & 262 & 0,21 \\
\hline & $\begin{array}{l}\text { Repetir em } 6 \\
\text { meses }\end{array}$ & 1.090 & 0,89 \\
\hline & Nova coleta & 137 & 0,11 \\
\hline \multirow{3}{*}{ Nova coleta } & $\begin{array}{l}\text { Seguir rotina de } \\
\text { rastreamento }\end{array}$ & 796 & 0,65 \\
\hline & $\begin{array}{l}\text { Repetir em } 6 \\
\text { meses }\end{array}$ & 82 & 0,07 \\
\hline & Colposcopia & 18 & 0,01 \\
\hline \multicolumn{2}{|l|}{ TOTAL } & 14.313 & 11,64 \\
\hline
\end{tabular}

\section{DISCUSSÃO}

Segundo a Organização Mundial da Saúde, a efetividade da detecção precoce do câncer do colo do útero por meio do exame de Papanicolaou associada ao tratamento em estágios iniciais pode reduzir em até $80 \%$ as taxas de incidência de câncer do colo do útero invasor, quando o rastreamento apresenta boa cobertura e é realizado dentro dos padróes de qualidade ${ }^{10}$.

A variabilidade interobservadores observada nos diagnósticos pelo método de Papanicolaou é relatada na literatura por se tratar de interpretaçáo subjetiva e com aplicação dos critérios diagnósticos descritos para cada categoria diagnóstica ${ }^{11-12}$. Essa variabilidade pode ser determinada através do kappa ponderado, preconizado para a avaliação de concordância atribuindo diferentes pesos às discordâncias diagnósticas ${ }^{6}$.

Este estudo mostrou concordância boa entre os lab-SUS e o LCO-IAL ( $k a p p a=0,77)$. A Unidade de Monitoramento Externo de Qualidade (UMEQ) do Paraná, ao revisar 65.753 casos, demonstrou concordância excelente entre os diagnósticos $(k a p p a=0,88)^{13}$. A UMEQ de Goiás estudou 10.053 casos e obteve concordância excelente ( $k a p p a=0,81)$ no período de 2007 a $2008^{14-15}$. Estes estudos foram realizados em condiçôes semelhantes, ou seja, com conhecimento prévio dos diagnósticos emitidos pelos laboratórios monitorados, resultando em alto índice de concordância entre os diagnósticos. Estudos realizados sem o conhecimento prévio do diagnóstico original refletem menor grau de concordância, como demonstrado por Stolerl e Schiffman, que obtiveram concordância moderada $(k a p p a=0,49)$ para diagnósticos de citologia líquida, nas categorias: negativo, ASCUS, LSIL e HSIL ${ }^{16}$.

A qualidade do exame citopatológico é condição fundamental para a garantia dos programas de rastreamento $^{17-18}$. Para melhorar a qualidade do exame citopatológico, são necessárias medidas de controle interno e externo da qualidade na rotina dos laboratórios. Os casos insatisfatórios e atipias de significado indeterminado podem ser utilizados como indicadores internos de qualidade nos laboratório ${ }^{8}$. Os relatórios do MEQ podem auxiliar os lab-SUS a identificar possíveis não conformidades, fornecendo subsídios para a implantação de estratégias de controle interno de qualidade e aprimoramento das preparações técnicas.

Os lab-SUS consideraram $7,6 \%$ das amostras insatisfatórias, sendo que, após a revisão, este percentual passou a ser de $12,5 \%$. Entre os diagnósticos insatisfatórios dos lab-SUS (7,6\%), foram detectadas alteraçôes epiteliais em $1,07 \%$. No período estudado, 16.581 (13,48\%) tiveram discordância diagnóstica, sendo que em $24,79 \%$ 
houve retardo da conduta adotada e em $22,11 \%$ houve prejuízo ao SUS por repetição desnecessária da citologia.

Estudos mostraram que os resultados FN são os responsáveis por cerca de $10 \%$ dos casos de câncer invasivo que progrediram a partir de lesóes pré-câncer não detectadas em testes anteriores ${ }^{19}$. Os resultados $\mathrm{FN}$ causam maior impacto aos programas de rastreamento, uma vez que as mulheres com diagnóstico $\mathrm{FN}$ poderâo ter perda de seguimento e permanecerão com risco de desenvolver o câncer do colo do útero ${ }^{1}$. Este estudo demonstrou que $1,76 \%$ dos resultados originalmente classificados como negativos apresentaram algum tipo de alteração epitelial quando submetidos à revisão, sendo que $1,52 \%$ dessas pacientes deveriam ter repetido a citologia em 6 meses, e $0,24 \%$ deveriam ter sido encaminhadas imediatamente para a colposcopia.

Ao se observar a especificidade (96,49\%) e sensibilidade (92,02\%) desses resultados, é importante salientar que, de acordo com as regras estabelecidas, todos os exames positivos e apenas $5 \%$ dos exames negativos são submetidos à revisão; fato que provavelmente reflete em subavaliação dos resultados FN. Para avaliação mais precisa do percentual, o procedimento mais adequado seria a revisão dos exames anteriores dos casos que apresentarem diagnóstico positivo atual, estratégia que deveria ser adotada pelos lab-SUS como ferramenta de controle interno da qualidade ${ }^{17}$.

Além das recomendaçôes básicas propostas pelo MS e INCA, o LCO-IAL introduziu, desde 2003, a avaliação pré-analítica e analítica de todas as amostras submetidas à revisão. São emitidos relatórios analítico e sintético, com descriçáo das condiçóes de acondicionamento e identificação das lâminas, da qualidade de fixação, coloração e confecção dos esfregaços, presença de contaminação, discordâncias diagnósticas e nomenclatura utilizada, com a finalidade de apontar aos lab-SUS quais os pontos críticos que interferem na concordância diagnóstica. A coloração e fixação das amostras são os pontos fracos dos lab-SUS, sendo que apenas 36,0\% e 43,0\% apresentam coloração e fixação adequadas, respectivamente.

Frente a essa realidade, organizaram-se cursos de reciclagem como ação corretiva, na tentativa de aprimorar o rastreamento pelo exame citopatológico cervical, com atualização e padronização de critérios diagnósticos, orientaçôes em procedimentos técnicos e utilização de ferramentas de controle de qualidade interno. A FOSP desenvolve programas de treinamento em colheita de amostras cervicovaginais para os profissionais das Unidades de Saúde. Durante o período avaliado, dez turmas participaram dos cursos de reciclagem, resultando no treinamento de 150 profissionais oriundos de 60
lab-SUS. Estudos futuros visam a avaliar qual o impacto da participação nos cursos de reciclagem e educação continuada para a melhoria da qualidade técnica e diagnóstica.

Em publicação anterior do nosso grupo, observou-se que, no período 2000 a 2004, houve aumento do número de lab-SUS que apresentaram concordância boa e excelente de acordo com o índice kappa, fato que atribui-se ao ajuste aos critérios diagnósticos ${ }^{20}$. Esse aspecto é relevante nas categorias diagnósticas que envolvem maior subjetividade como nas atipias de significado indeterminado e na avaliação de satisfatoriedade das amostras. Moore et al. ${ }^{21}$ afirmaram que o treinamento melhora a concordância interobservador em relaçáo à satisfatoriedade das amostras citológicas. Sebastiāo et al. ${ }^{22}$, ao analisarem a categoria de atipias de significado indeterminado na UMEQ do Paraná, observaram que o MEQ pode contribuir para a melhoria desses diagnósticos.

\section{CONCLUSÃO}

Conclui-se que o MEQ é parte da estratégia dos programas de rastreamento organizados, sendo uma ferramenta de educação continuada que contribui para a uniformização dos critérios citomorfológicos e melhoria do desempenho diagnóstico, refletindo na redução dos resultados FN e FP. No entanto, o MEQ não é amplamente implantado no país.

Além do conjunto de atividades básicas propostas pelo MS, a equipe do MEQ do Estado de SP traz inovaçóes ao modelo, com a intenção de aumentar a eficácia das açóes de controle de qualidade dos exames citopatológicos. Ainda que se tenha apontado as deficiências e sugerido medidas corretivas para melhoria da qualidade da citologia nos lab-SUS, alguns problemas detectados são recorrentes e perduram. Isto se deve, em parte, ao fato de o MEQ em SP ter caráter educativo. Para o sucesso de qualquer programa de qualidade, é necessária a atuação de uma coordenação que assuma a tarefa de fiscalizar a implementaçáo das medidas corretivas.

Este estudo, além de apresentar os dados relativos aos percentuais de discordância diagnóstica, mostra os principais desafios enfrentados ao longo de mais de uma década de experiência no programa de MEQ. Acredita-se que a troca de experiência é de fundamental importância, visto que, atualmente, o MS vem incentivando a disseminação das UMEQ em todos os estados do Brasil.

\section{AGRADECIMENTOS}

Aos profissionais do Instituto Adolfo Lutz, Camilo de Lelis Feres, Rosemeire Rodrigues de Oliveira Lima e Neuza 
Kasumi Shirata, pela participação na revisão das lâminas; Rosângela Santos de Araújo, Rosana Astoni Ambrus e Vera Lúcia Moreno Silva, pela participação na triagem, digitação e recepção do material. À Lise Cristina P. Baltar Cury e aos demais profissionais envolvidos no suporte administrativo da Fundação Oncocentro de São Paulo.

\section{CONTRIBUIÇÕES}

Participaram da concepção e planejamento Daniela Etlinger, Sonia Maria Miranda Pereira e Celso di Loreto. Participaram da obtenção e/ou análise e interpretação dos dados Daniela Etlinger, Yuriko Ito Sakai, Sonia Maria Miranda Pereira, Luzia Setuko Umeda Yamamoto e Celso di Loreto. Participaram da revisão crítica Daniela Etlinger, Sonia Maria Miranda Pereira, Silvia D’Andretta Iglezias, Monique Camila Basso e Celso di Loreto.

\section{Declaraçáo de Conflito de Interesses: Nada a Declarar.}

\section{REFERÊNCIAS}

1. Monsonego J, Bosch FX, Coursaget P, Cox JT, Franco E, Frazer I, et al. Cervical cancer control, priorities and new directions. Int J Cancer. 2004;108(3):329-33.

2. Instituto Nacional de Câncer José Alencar Gomes da Silva. Câncer do colo do útero [Internet]. [citado 2011 jul 11]. Disponível em: http://www2.inca.gov.br/wps/ $\mathrm{wcm} /$ connect/tiposdecancer/site/home/colo_utero

3. Bernstein SJ, Sanchez-Ramos L, Ndubisi B. Liquidbased cervical cytologic smear study and conventional Papanicolaou smears: a metaanalysis of prospective studies comparing cytologic diagnosis and sample adequacy. Am J Obstet Gynecol. 2001;185(2):308-17.

4. Cohn DE, Herzog TJ. New innovations in cervical cancer screening. Clin Obstet Gynecol. 2001;44(3):538-49.

5. São Paulo. Resolução SS-116, de 27 de julho de 2000. Dispóe sobre o Programa de Controle de Qualidade em Exames Colpocitológicos para prevenção e detecção do câncer do colo uterino e lesões precursoras no âmbito do SUS/SP. Diário Oficial do Estado de São Paulo, São Paulo (2000 jul 28);Sec.1:34.

6. São Paulo. Fundação Oncocentro de São Paulo. Portaria FOSP 10, de 12 de setembro de 2000. Dispõe sobre a operacionalização do Programa de Controle de Qualidade em Exames Colpocitológicos. Diário Oficial do Estado de São Paulo, São Paulo (2000 set 13);Sec.1:17.

7. Brasil. Secretaria de Assistência à Saúde. Portaria conjunta n. 92 de 16 de outubro de 2001. Art. 3º. Determinar a execução do monitoramento interno da qualidade dos resultados de exames citopatológicos, por parte de todo laboratório que realize esses exames para o Sistema Único de Saúde-SUS. Diário Oficial da União, Brasília (2001 out 17);Sec.1:55.
8. Instituto Nacional de Câncer (Brasil). Nomenclatura brasileira para laudos cervicais e condutas preconizadas: recomendações para profissionais de saúde. J bras patol med lab. 2006;42(5):351-73.

9. Instituto Nacional de Câncer (Brasil). Viva Mulher. Câncer do colo do útero: informações técnico-gerenciais e ações desenvolvidas. Rio de Janeiro: INCA; 2002.78 p.

10. World Health Organization. Manual on the prevention and control of common cancers. Geneva: WHO; 1998. 318 p. (WHO regional publications. Western Pacific series; n. 20).

11. Tuon FFB, Bittencourt MS, Panichi MA, Pinto AP. Avaliação da sensibilidade e especificidade dos exames citopatológico e colposcópico em relação ao exame histológico na identificação de lesōes intra-epiteliais cervicais. Rev Assoc Med Bras. 2002;48(2):140-4.

12. Vooijs GP, Nauwelaers FA, van Aspert-van Erp AJ. CytosafePLUS: a workstation for screening, supervision, reviewing, quality assurance and education in cytopathology. Acta Cytol. 1996;40(1):90-6.

13. Collaço LM, Noronha L, Bleggi-Torres LF, Pinheiro DL. Quality control in cervical cancer screening: Brazilian experience. Acta Cytol. 2005;49(6):694-6.

14. Ázara CZS. Avaliação dos resultados dos exames citopatológicos do colo do útero dos laboratórios credenciados pelo Sistema Único de Saúde de acordo com o diagnóstico da Unidade de Monitoramento Externo da Qualidade [dissertação]. Goiânia: Universidade Federal de Goiás; 2010.

15. Amaral RG, Souza NLA, Tavares SBN, Manrique EJC, Assem DZ, Azevedo LL, et al. Controle externo da qualidade dos diagnósticos citológicos no rastreamento do câncer cervical: estudo piloto. Rev bras anal clin. 2006:38(2):79-81.

16. Stoler MH, Schiffman M; Atypical squamous cells of undetermined significance-low-grade squamous intraepithelial lesion triage study (ALTS) Group. Interobserver reproducibility of cervical cytologic and histologic interpretations: realistic estimates from the ASCUS-LSIL Triage Study. JAMA. 2001;285(11):1500-5.

17. Kostova P. [Cervical screening: its essence and qualitycontrol methodology in cytology laboratories]. Akush Ginekol (Sofiia). 1998;37(4):58-61. Bulgarian.

18. Flisser A, García-Malo F, Canepa MLA, Doncel S, Espinoza R, Moreno R, et al. Implementation and evaluation of a national external quality control program for cervical cytology in Mexico. Salud pública Méx. 2002;44(5):431-6.

19. Pittoli JE, Mello ES, Pereira SMM, Maeda MYS, Utagawa ML, Celestino JD, et al. Revisão de esfregaços cervicais negativos em pacientes com lesões intra-epiteliais de alto grau. J bras patol med lab. 2003;39(3):219-21.

20. Pereira SMM, Ramos DEL, Yamamoto LSU, Shirata NK, Loreto C, Ferraz MGMC, et al. Monitoramento externo de qualidade em citopatologia cervical e o reflexo 
na rotina dos laboratórios da rede pública. DST $\mathrm{j}$ bras doenças sex transm. 2006;18(3):172-7.

21. Moore D, Pugh-Cain D, Walker T. Cervical smear adequacy: cellularity references were found to increase both interobserver agreement and unsatisfactory rate. Cytopathology. 2009;20(3):161-8.
22. Sebastião APM, Noronha L, Scheffel DLH, Garcia MJ, Carvalho NS, Collaço LM, et al. Estudo das atipias indeterminadas em relação à prevalência e ao percentual de discordância nos casos do Programa de Prevenção do Câncer Uterino do Paraná. J bras patol med lab. 2004;40(6):431-8. 


\begin{abstract}
Introduction: The External Quality Assurance Program of cervical cytophatology exams carried out by the Brazilian Unified Health System was proposed by the Ministry of Health to improve the performance of cytology laboratories of the Public Health Network. Objective: To evaluate the disagreement between the diagnoses of cytology laboratories and the revision by the External Quality Assurance Program in the state of São Paulo, from 2000 to 2010. Method: Retrospective comparison of the original cytologic diagnoses issued by the Public Health Network Laboratories with the revision of the cervical cytophatology exams. Results: Of the 123,002 samples reviewed, 16,581 (13.48\%) had diagnostic disagreement. In 14,313 (11.64\%) of them, there was a divergence between the clinical approach used and that advocated by the Brazilian National Cancer Institute - José Alencar Gomes da Silva (INCA). False-positive samples were 2,530 (2.06\%) and 2,154 were false-negative (1.75\%). The sensitivity was $92.02 \%$ and specificity was $96.49 \%$. Kappa index showed good agreement (0.77) between the original and the revised diagnoses. Conclusion: Besides the set of basic activities proposed by the Ministry of Health, the External Quality Assurance Program of the state of São Paulo highlights the continuing educational activities that were implemented, which were adopted to promote the continuing education towards the standardization of cytomorphological criteria and the consequent reduction of false-negative and false-positive results.

Key words: Quality Control; Cytodiagnosis; Uterine Cervical Neoplasms/diagnosis; Uterine Cervical Neoplasms/ pathology; Mass Screening; Cervix Neoplasms Prevention
\end{abstract}

\footnotetext{
Resumen

Introducción: El programa de Supervisión Externa de Calidad de las pruebas citopatológicas cervico-vaginales realizadas por el Sistema Único de Salud fue planteado por el Ministerio de Salud para mejorar el rendimiento de los laboratorios de citología de la Red Pública de Salud. Objetivo: Evaluar las discrepancias diagnósticas de las pruebas citopatológicas cervico-vaginales sometidas a la revisión por el Programa de Supervisión Externa de Calidad del Estado de São Paulo en el período 2000 hasta 2010. Método: Evaluación por comparación retrospectiva de los diagnósticos emitidos por los laboratorios SUS y de los diagnósticos de revisión de las pruebas citopatológicas cervico-vaginales. Resultados: De las 123.002 muestras repasadas, 16.581 (13,48\%) presentaban discordancia diagnostica, y en 14.313 $(11,64 \%)$ casos hubo una divergencia entre el enfoque adoptado y el sugerido por el Instituto Nacional del Cáncer José Alencar Gomes da Silva (INCA). Las pruebas han sido consideradas falsos positivos 2.530 (2,06\%) y falsos negativos 2154 (1,75\%). La sensibilidad fue del 92,02\% y una especificidad del 96,49\%. Hubo buena concordancia entre los diagnósticos originales y de revisión (kappa=0,77). Conclusión: Además del conjunto de actividades básicas propuestas por el Ministerio de Salud, el Programa de Supervisión Externa de Calidad del Estado de Sáo Paulo destaca las acciones educativas implementadas, adoptadas para promocionar la educación continuada dirigida a la similitud de los criterios citomorfológico y la consecuente reducción de los falsos negativos y falsos positivos.

Palabras clave: Control de Calidad; Citodiagnóstico; Neoplasias del Cuello Uterino/diagnóstico; Neoplasias del Cuello Uterino/patología; Tamizaje Masivo; Prevención de Cáncer de Cuello Uterino
} 